ARTICLE

DOI: $10.1038 / s 41467-017-02708-5$

\title{
Genome-wide tracking of dCas9-methyltransferase footprints
}

Christina Galonska', Jocelyn Charlton 1,2, Alexandra L. Mattei (10) 1,2,3, Julie Donaghey 2,3, Kendell Clement (10) 2,3, Hongcang Gu${ }^{3}$, Arman W. Mohammad ${ }^{3}$, Elena K. Stamenova ${ }^{3}$, Davide Cacchiarelli ${ }^{3,7}$, Sven Klages ${ }^{1}$, Bernd Timmermann ${ }^{1}$, Tobias Cantz ${ }^{4}$, Hans R. Schöler ${ }^{5}$, Andreas Gnirke ${ }^{3}$, Michael J. Ziller ${ }^{6}$

\& Alexander Meissner ${ }^{1,2,3}$

In normal mammalian development cytosine methylation is essential and is directed to specific regions of the genome. Despite notable advances through mapping its genome-wide distribution, studying the direct contribution of DNA methylation to gene and genome regulation has been limited by the lack of tools for its precise manipulation. Thus, combining the targeting capability of the CRISPR-Cas9 system with an epigenetic modifier has attracted interest in the scientific community. In contrast to profiling the genome-wide cleavage of a nuclease competent Cas9, tracing the global activity of a dead Cas9 (dCas9) methyltransferase fusion protein is challenging within a highly methylated genome. Here, we report the generation and use of an engineered, methylation depleted but maintenance competent mouse ES cell line and find surprisingly ubiquitous nuclear activity of dCas9methyltransferases. Subsequent experiments in human somatic cells refine these observations and point to an important difference between genetic and epigenetic editing tools that require unique experimental considerations.

\footnotetext{
${ }^{1}$ Department of Genome Regulation, Max Planck Institute for Molecular Genetics, 14195 Berlin, Germany. ${ }^{2}$ Department of Stem Cell and Regenerative Biology, Harvard University, Cambridge, MA 02138, USA. ${ }^{3}$ Broad Institute of MIT and Harvard, Cambridge, MA 02142, USA. ${ }^{4}$ Translational Hepatology and Stem Cell Biology, Hannover Medical School, Hannover 30625, Germany. ${ }^{5}$ Department of Cell and Developmental Biology, Max Planck Institute for Molecular Biomedicine, Münster 48149, Germany. ${ }^{6}$ Department for Translational Psychiatry, Max Planck Institute of Psychiatry, Munich 80804, Germany. ${ }^{7}$ Present address: Armenise-Harvard Laboratory of Integrative Genomics, Telethon Institute of Genetics and Medicine (TIGEM), Pozzuoli 80078, Italy. Christina Galonska and Jocelyn Charlton contributed equally to this work. Correspondence and requests for materials should be addressed to A.M. (email: meissner@molgen.mpg.de)
} 
D NA methylation is widespread among organisms, with the core enzymes that catalyze the methyl group transfer being conserved for more than a billion years across plants and animals ${ }^{1-3}$. Comparative genome-wide DNA methylation mapping has enhanced our understanding of the mammalian targets and dynamics of this modification ${ }^{2,4-7}$, but many important questions regarding its precise regulatory role remain unanswered. The complex multilayered mechanisms by which DNA methylation is regulated and mitotically maintained complicate its study and the absence of tools that enable targeted manipulation has limited progress further.

However, recent advances in the field of genome editing have raised hopes that these technical limitations may finally be overcome $^{8}$. In particular, the CRISPR-Cas9 system for genome engineering has emerged as a powerful genomics toolbox due to its high targeting specificity and efficiency ${ }^{9}$. More recently, fusion of effector domains or proteins to the catalytically inactive (dead) dCas9 protein extended the potential applications to targeted epigenome editing $^{9-16}$, including de novo methylation through dCas9-methyltransferase fusion proteins. However, several critical questions need to be explored before DNA methylation editing can be considered a reliable tool. It remains unclear what characteristics render a given locus susceptible to become ectopically methylated, i.e. how much does the transcriptional or chromatin state of a given target matter? Can canonically unmethylated regions be targeted and the methylation maintained in the absence of the inducer? For instance, recent studies suggest that directed methylation can alter target gene expression, although methylation is rapidly lost upon removal of the dCas9effector $^{12,13,17}$. How dependent is the dCas9-methyltransferase on the presence of the endogenous de novo machinery? Finally, how much off-target activity arises when the dCas9-methyltransferase complex is present in the nucleus near its substrate (all cytosines)?

As for the latter, previous studies show that the nuclease active Cas9 rarely cuts at off-target sites, despite widespread engagement as shown by genome-wide mapping ${ }^{18}$. However, chromatin immunoprecipitation (ChIP)-based approaches are not sensitive enough to detect transient or past interactions, which may be sufficient to induce lasting epigenetic alterations such as DNA methylation. Furthermore, high levels of DNA methylation and the presence of the endogenous de novo DNA methyltransferases (Dnmts) complicate any accurate evaluation of dCas9methyltransferase activity in the nucleus ${ }^{5,7}$. Limited by these factors, current literature offers preliminary insights into the general applicability and on-target methylation efficiency of dCas9-fused methylation effectors yet lacks a general interpretation of global off-target activity. The same drawbacks have also restricted the precise measurement of seeding, spreading and maintenance of targeted DNA methylation. Here, we present a system to measure several of these parameters and explore the effects of dCas9-methyltransferases in pluripotent cells. We observe widespread off-target activity of dCas9-methyltransferases, which occurres independently of the presence of single guide RNAs (sgRNAs) and was also apparent across multiple somatic cell types. Our results therefore provide valuable insight into the utility of epigenome editing tools that should be considered in future experimental designs.

\section{Results}

Generation of an ES cell model to track de novo methylation. To systematically assess the global effects of dCas9-fused methyltransferases, we used previously established $D n m t 3 a / b$ double knockout (DKO) embryonic stem (ES) cells ${ }^{19}$ and transiently repressed the maintenance methyltransferase Dnmt1 to deplete global methylation levels (as described previously ${ }^{20}$ ). This strategy allowed us to derive a new cell line (termed $\mathrm{DKO}^{\text {zero }}$ ) that has significantly reduced cytosine methylation (mean global CpG methylation measured by reduced representation bisulfite sequencing (RRBS)/ whole-genome bisulfite sequencing (WGBS): wild-type ES cells $0.57 / 0.83$ and $\mathrm{DKO}^{\text {zero }}$ : $0.04 / 0.04$ ) but is able to maintain any emerging DNA methylation through the reexpression of endogenous Dnmt1 (Fig. 1a, b, Supplementary Fig. 1a, b; Methods section). As a reference point, the global CpG methylation levels in the $\mathrm{DKO}^{\text {zero }}$ cell line are below what has been observed for wild-type ES cells grown in $2 \mathrm{i} / \mathrm{LIF}$ conditions ${ }^{21}$ (Supplementary Fig. 1a). Continued passaging of the $\mathrm{DKO}^{\text {zero }}$ cells did not result in any notable gain of global DNA methylation, suggesting limited de novo activity of DNMT1 (Fig. 1c, Supplementary Fig. 1c). As a result, these DNA methylation depleted, maintenance competent ES cells allow us to precisely track the extent of newly added DNA methylation (without interference from the endogenous de novo enzymes) including past engagements of the catalytic domain with unmethylated cytosines that are subsequently propagated by DNMT1.

dCas9-cat3a displays global nuclear activity. After we established our $\mathrm{DKO}^{\text {zero }}$ system, we generated a doxycycline (Dox) responsive construct that when co-introduced with the reverse trans-activator (M2rtTA) enables the inducible expression of dCas9-cat3a (dCas9 fused to the catalytic domain of Dnmt3a; Fig. 1d). After viral transduction and selection for double positive cells (M2rtTA plus the inducible construct), we derived a clonal cell line (DKO ${ }^{\text {dCas9-cat3a) }}$ ) and assessed dCas9-cat3a induction by measuring GFP expression using fluorescence activated cell sorting (FACS). Based on this analysis we observed homogenous induction in the majority of cells (Supplementary Fig. 1d) with methylation analysis confirming no detectable leakiness in the absence of Dox (Supplementary Fig. 1e). However, we noticed to our surprise that the transient induction with Dox had already led to widespread gain of $\mathrm{CpG}$ methylation (Supplementary Fig. 1f, left), suggesting strong global activity by dCas9-cat3a. To measure the impact of dCas9-cat3a induction more precisely, we reset global methylation in the $\mathrm{DKO}^{\mathrm{dCas} 9-\mathrm{cat} 3 \mathrm{a}}$ line through another round of transient Dnmt1 depletion as well as transient culture in 2i/LIF plus Vitamin C, a combination that rapidly reduces global DNA methylation ${ }^{22}$. After again confirming the global depletion of DNA methylation (Supplementary Fig. 1f, right), we induced the reset $\mathrm{DKO}^{\mathrm{dCas} 9-\mathrm{cat} 3 \mathrm{a}}$ cell line in the absence of a sgRNA for 0 (uninduced control), 2 and 7 days and performed WGBS (Fig. 1e, f). This data not only confirmes our prior observation but also highlights how rapid and widespread the increase in DNA methylation occurs (mean CpG methylation uninduced: 0.16; 2 days: 0.28 and 7 days: 0.47 ). Upon closer inspection, we found that the gain of methylation is largely defined by the prior methylation state in wild-type ES cells. Specifically, genomic features that were highly methylated in wild-type ES cells, such as exons and repetitive elements, showed a notable gain upon Dox induction, as did regions of intermediate methylation (mean $>0.2$ and $<0.8$ in wild-type cells) that represent putative enhancer elements with enrichment for H3K27ac (Fig. 1g, Supplementary Fig. 1g). Alternatively, default unmethylated sites, such as $\mathrm{CpG}$ islands (CGIs) associated with transcription start sites, remained generally lowly methylated (Fig. 1g). A smaller subset of CGIs that were methylated in wild-type ES cells (mean $>0.8, n=2160$ / 21,012) showed a strong gain to 0.56 mean methylation in $\mathrm{DKO}^{\text {dCas9-cat3a }}$ cells after 7 days, which may be linked to the lack of H3K4me3 (Fig. 1h, left). Supporting this, CGIs that were unmethylated in wild-type ES cells and remained so in the induced cells $(n=13,525$, mean methylation $=0.06$ and 0.09 for 
a

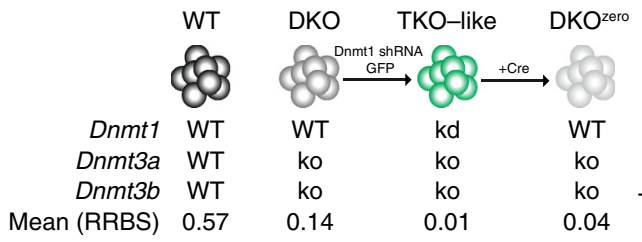

b

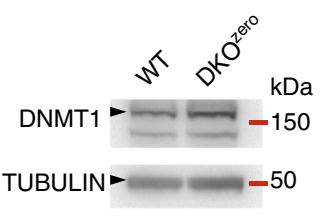

d

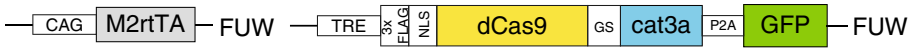

C

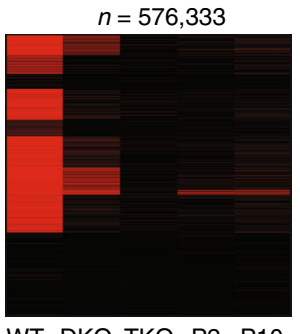

WT DKO TKO- P2 P10 like DKO zero

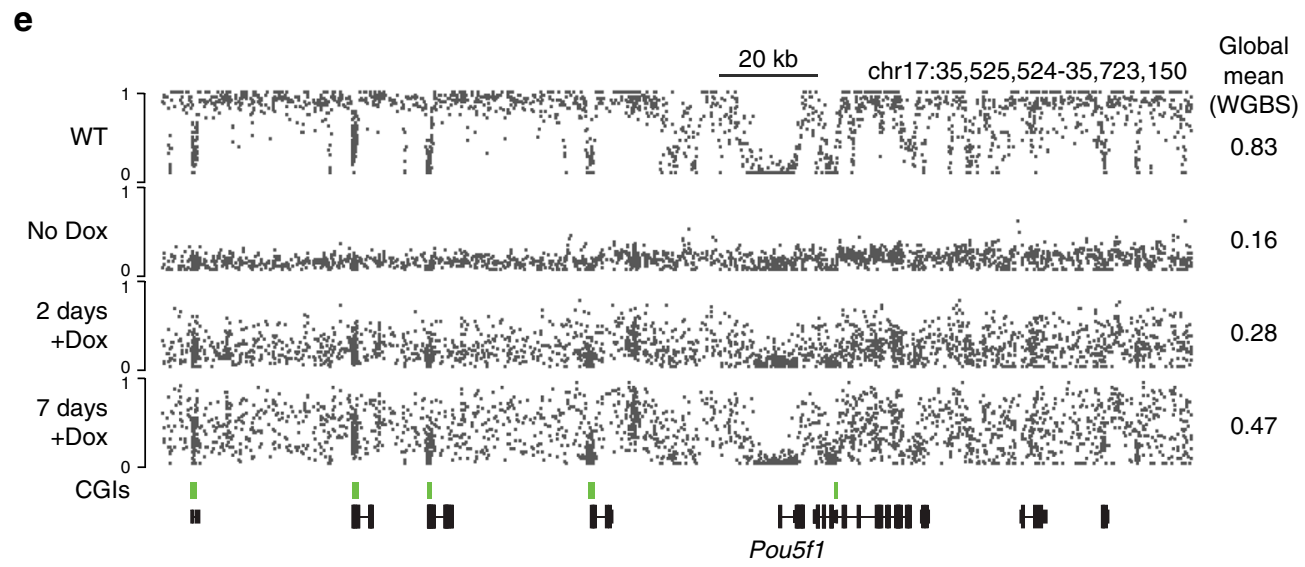

f

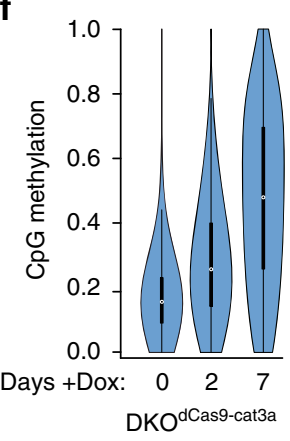

g

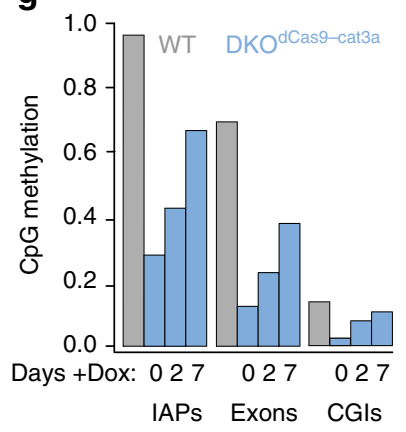

h

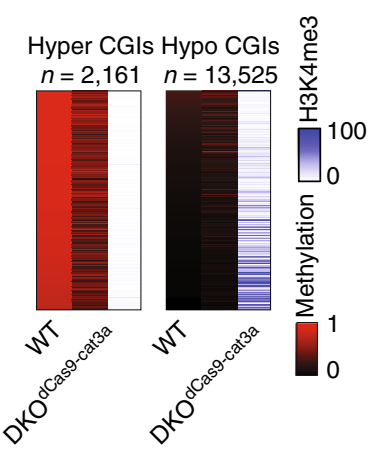

Fig. 1 Characterization of dCas9-cat3a activity in a methylation depleted cell line. a Overview of the multi-step generation of the Dnmt3 (de novo) free, maintenance competent, methylation depleted DKOzero ES cell line (see Methods section for details), WT = wild-type, $k d=k n o c k$ down, $k o=k n o c k o u t$. Mean = mean methylation for matched CpGs measured by reduced representation bisulfite sequencing (RRBS). b Western blot of DNMT1 (183 kDa) in WT and DKO ${ }^{z e r o}$ cells. TUBULIN ( $50 \mathrm{kDa}$ ) serves as loading control. c Heatmap comparing global CpG methylation levels measured by RRBS in WT, DKO, TKO-like, early (P2) and late (P10) passage DKOzero cells. $n$ shows the number of shared CpGs $(n=576,333)$. d Simplified schematics showing the M2rtTA and dCas9-cat3a FUW-based lentiviral expression constructs. e Representative whole genome bisulfite sequencing (WGBS) tracks displaying CpG methylation in WT as well as reset DKOdCas9-cat3a cells with 0,2 , or 7 days of Dox induction. Global mean CpG methylation levels are shown on the right. CpG islands (CGIs) are highlighted beneath in green. $\mathbf{f}$ Violin plots for matched CpGs in DKOdCas9-cat3a with 0, 2, and 7 days of Dox induction without the presence of an sgRNA. White dots indicate median values. $\mathbf{g}$ Mean $\mathrm{CpG}$ methylation across selected features in WT and DKO dCas9-cat3a cells with 0 , 2, and 7 days of Dox induction. IAPs = intra-cisternal A-type particles (retrotransposons). $\mathbf{h}$ Heatmap for all hypermethylated CGls (mean in WT $>0.8$, left) and

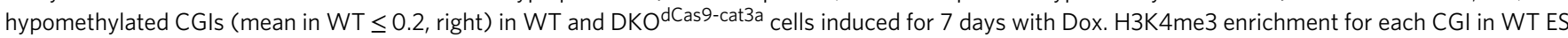
cells is displayed

wild-type and 7 day-induced $\mathrm{DKO}^{\mathrm{dCas} 9-\mathrm{cat3a}}$, respectively) showed greater enrichment for H3K4me3. Nonetheless, a subset of hypomethylated CGIs gained a small, but significant $(p<0.01)$ amount of DNA methylation (Fig. 1h, right, Supplementary Fig. 1h, i). Finally, when we introduced the dCas9-cat3a into normally methylated, wild-type ES cells we also observed overall protection of hypomethylated CGIs (Supplementary Fig. 1j) with little overall increase due to the already high level of genomic methylation (Supplementary Fig. 1k). These results are in agreement with the previously noted association of methylated histone $\mathrm{H} 3 \mathrm{~K} 4$ tails with protection of CGIs from the de novo DNA methylation machinery ${ }^{23,24}$, which we extend here to cells with ectopic methyltransferase activity.

Ubiquitous nuclear activity is unaffected by sgRNA presence. To explore if the addition of one or more sgRNAs would 
constrain the ubiquitous nuclear activity of the dCas9-cat3a to selected target loci, we stably integrated sgRNAs into our $\mathrm{DKO}^{\mathrm{dCas} 9-\text { cat3a }}$ cell line and compared on- and off-target methylation to our no guide controls. First, we tested one sgRNA against the Dazl promoter, a highly methylated, lowly expressed locus in wild-type ES cells with reduced CpG methylation in our $\mathrm{DKO}^{\mathrm{dCas} 9-\mathrm{cat} 3 \mathrm{a}}$ cell line. We independently validated the targeting efficiency of this sgRNA through the transient expression of an active Cas9 enzyme, and observed efficient cutting that mapped to the expected target site (Supplementary Fig. 2a). Interestingly, the addition of the Dazl sgRNA prior to Dox induction did not prevent the global gain of CpG methylation when compared to the no guide condition (Supplementary Fig. 2b). Moreover, while we observed a gain of $\mathrm{CpG}$ methylation around the sgRNA target sequence, this increase also occurred independently of the sgRNA, making it difficult to quantify the actual on-target activity for this locus (Supplementary Fig. 2c). To explore the possibility of multi-loci targeting and to see whether additional guides would help reduce the off-target activity, we next transduced our DKO ${ }^{\text {dCas9-cat3a }}$ cell line with a pool of ten sgRNAs that target representative loci with distinct methylation and expression levels in wild-type ES cells (Fig. 2a, Supplementary Fig. 3a). We used universal primers for the backbone to confirm the presence of sgRNAs via amplicon sequencing in several clonal lines (see Methods section) and initially focused on clone 3 in which eight of the ten sgRNAs were integrated. To assess methylation at our regions of interest with high coverage, we designed a specific set of $120 \mathrm{bp}$ hybrid capture probes for targeted bisulfite sequencing, tiling $20 \mathrm{~kb}$ on either side of the sgRNA target. This strategy provides high-resolution information of the target locus and a representative bidirectional genomic sampling surrounding it (Fig. 2a; Supplementary Fig. 3b, c; Supplementary Table 1). Given the rapid rate of methylation gain initially observed, we chose earlier time points and collected samples 1, 2 and 3 days post Dox treatment as well as an uninduced (0 days) control (Fig. $2 \mathrm{a}-\mathrm{c}$ ). Similar to our prior observations, off-target methylation accumulated along each captured region (Fig. 2b, c; Supplementary Fig. 3c), demonstrating that even multiple sgRNAs are not sufficient to prevent dCas9-cat3a from acting off-target. Of interest, we did observe on-target methylation at the Foxb1 locus, which is unmethylated and not expressed in wild-type ES cells, while other sites such as the also unmethylated but highly expressed $I d 3$ locus did not accumulate target methylation above background (Fig. 2c, d; Supplementary Fig. 3c). It therefore appears that the on-target activity is somewhat context dependent and the expression status may have some impact on how susceptible a locus is to methylation gain in response to dCas9-cat3a recruitment. Overall, on-target vs. offtarget methylation accumulated faster at only two out of six loci (Fig. 2d). This was consistent across three independent clones which showed a reproducible global gain of methylation across all captured tiles (Fig. 2e). Notably, although mean off-target methylation was comparable for all $\mathrm{CpGs}$, we found variability in the affected regions between the clones (Fig. 2f), which may suggest a largely random off-target activity at accessible sites. Taken together, these results show that the presence of sgRNAs can increase on-target methylation, but has no detectable impact on reducing global dCas9-cat3a off-target activity.

Catalytic domain of Dnmt3a alone exhibits similar activity. Given these results, it was important to determine whether offtarget methylation was mediated by dCas9 scanning the DNA independently of an sgRNA ${ }^{25}$ or related to the unspecific activity and ubiquitous nuclear presence of the catalytic domain of Dnmt3a (cat3a) itself. To explore this we generated two additional inducible cell lines, one expressing only the Dnmt3a catalytic domain $\left(\mathrm{DKO}^{\mathrm{cat} 3 \mathrm{a}}\right)$ and a second one expressing the fulllength Dnmt3a (DKO ${ }^{\text {Dnmt3a }}$, that includes both cat3a and its $\mathrm{N}$ terminal regulatory domain; Supplementary Fig. 4a). After around two weeks of induction, $\mathrm{DKO}^{\text {Dnmt3a }}$ cells showed near wild-type levels of $\mathrm{CpG}$ methylation, while $\mathrm{DKO}^{\text {cat3a }}$ and $\mathrm{DKO}^{\mathrm{dCas} 9-\mathrm{cat} 3 \mathrm{a}}$ cells reached only intermediate levels (Supplementary Fig. 4b, c). Although mechanistic details remain to be elucidated, the different remethylation behaviors may result from reduced de novo activity and/or less efficient inheritance in the absence of the N-terminal regulatory domain of Dnmt3a. Furthermore, despite the fact that in principle any cytosine is a potential target for de novo methylation, we found that only cytosines in the $\mathrm{CpG}$ context were methylated in $\mathrm{DKO}^{\mathrm{dCas} 9-\text { cat3a }}$ and $\mathrm{DKO}^{\text {cat3a }}$ cells (Supplementary Fig. $4 \mathrm{~d}$, e). In contrast, the $\mathrm{DKO}^{\text {Dnmt3a }}$ cells showed methylation of cytosines in both the $\mathrm{CpG}$ and $\mathrm{CpA}$ context, the latter being the most frequently methylated non-CpG dinucleotide ${ }^{26}$. This suggests a possible dependence on the Dnmt3a regulatory domain for the acquisition of non-CpG methylation.

dCas9-cat3a induces off-target methylation in somatic cells. While our inducible ES cell lines have the advantage of homogenous induction and temporal control, most prior studies have selected transient transfection as the method of choice for introducing dCas 9 epigenetic effectors. Therefore, we decided to compare the consequences of a transient transfection strategy ${ }^{13}$ in our methylation depleted, maintenance competent $\mathrm{DKO}^{\text {zero }}$ system (Supplementary Fig. 5a) as well as in two frequently utilized human somatic cell lines (293T; human embryonic kidney and MCF7; human breast adenocarcinoma, Fig. 3a). Consistent with the results of the inducible line, we found a global gain of CpG methylation (and lack of non-CpG methylation) when transiently transfecting our $\mathrm{DKO}^{\text {zero }}$ line (Supplementary Fig. 5a-c). Importantly, no gain of methylation was observed when the cells were transfected with a control construct encoding a mutated (E756A), catalytically inactive version of cat3a (dCas9-cat3aANV $^{13}$, Supplementary Fig. 5d, e). For the 293T and MCF7 cells we utilized a previously described $B A C H 2$ sgRNA and observe on-target methylation that is comparable to their published results $^{13}$ (Fig. 3b, c). Of note, BACH2 is either not or only marginally expressed in both of our somatic cell lines (FPKM 293T: 1.11, FPKM MCF7: < 1). This low expression may render the locus more amenable to targeted methylation, similar to the Foxb1 locus in the mouse ES cells (Fig. 2b). Despite successful ontarget methylation, we observed an extensive accumulation at offtarget sites (Fig. 3d, e). Notably, both cell lines are globally methylated-unlike our $\mathrm{DKO}^{\text {zero }}$ line-and therefore make it more difficult to determine the exact scale of the off-target activity. When we compared $200 \mathrm{bp}$ tiles between the dCas9cat3a-ANV control and the dCas9-cat3a, 91\% (MCF7) and 94\% (293T) of the significantly different tiles (adj. $p$-value $<0.05(t$ test) and difference in methylation $>0.1$ ) overlapped with otherwise lowly methylated CGIs (Fig. 3d-f), with targets varying between 293T and MCF7 cells (Supplementary Fig. 5f). Biological replicates showed a similar behavior, with 50\% (293T Rep1) and 70\% (293T Rep2) significant tiles being shared targets, indicating that dCas9-cat3a is acting genome-wide with no obvious sequence preference (Supplementary Fig. 5g). To more closely inspect the gain of methylation, we performed read-level analysis and find that for both on- and off-target sites only half of the reads show any methylation (Supplementary Fig. 5h, i), suggesting either a mixed population of cells or that the de novo methylation is added to only one strand generating hemimethylated DNA. In agreement with previous reports we 
a

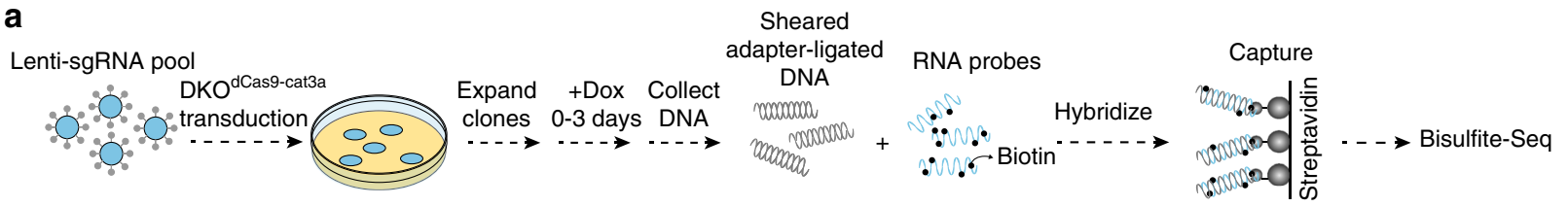

b

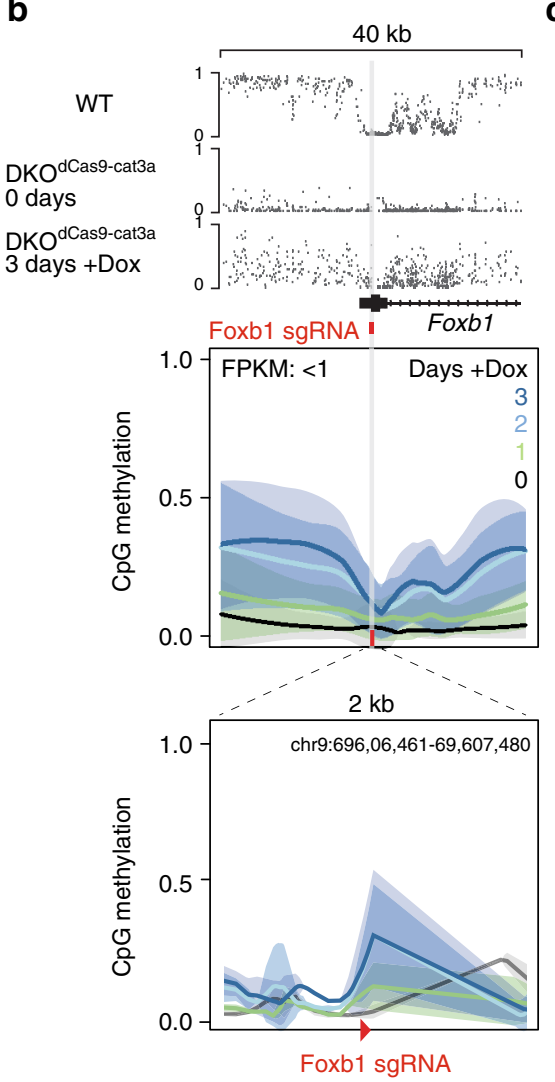

C

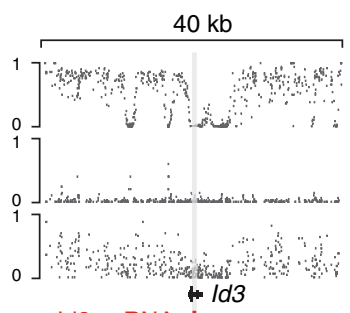

Id3 sgRnA ।

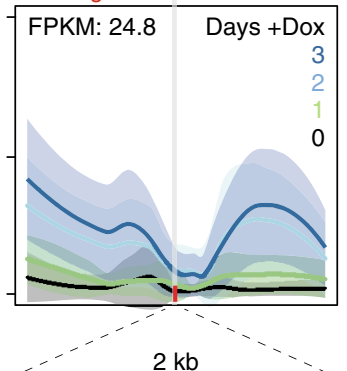

chr4:135,698,940-135,700,959

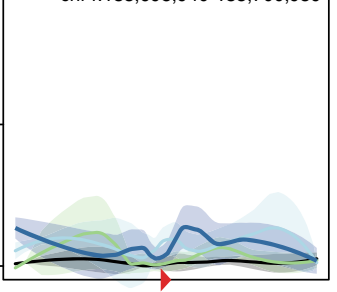

Id3 sgRNA d

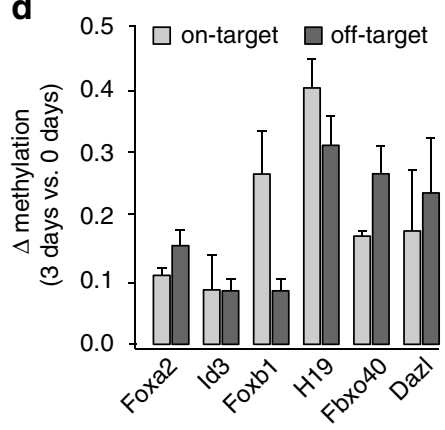

e

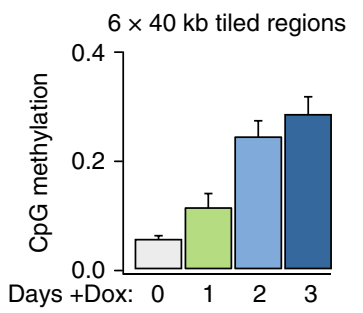

f

Top 100 methylated tiles

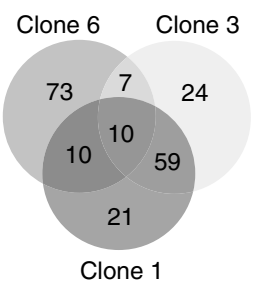

Fig. 2 Targeted bisulfite sequencing surrounding multiple sgRNA targets. a Schematic describing the experimental design for the transduction of DKOdCas9-cat3a cells with multiple sgRNA constructs. Cells were collected at 0, 1, 2 and 3 days after Dox treatment. Extracted DNA was hybridized to capture probes for enrichment of target loci. See Methods section for more details. b Top: tracks displaying methylation levels for wild-type (WT) ES cells and DKOdCas9-cat3a at 0 and 3 days of Dox treatment. The target site for the Foxb1 sgRNA is displayed with $20 \mathrm{~kb}$ on either side, captured by our custom probes. Below: loess-smoothed methylation levels for 0, 1, 2 and 3 days of Dox treatment for the full region shown at the top, as well as a zoomed in region displaying the $2 \mathrm{~kb}$ surrounding the targeted locus. The sgRNA position is indicated as a red bar in the top part and red arrowhead in the bottom zoom in. $\mathbf{c}$ Same as in $\mathbf{b}$, but for the Id3 locus. $\mathbf{d}$ The difference in methylation after 3 days of dCas9-cat3a expression for the on-target locus (200 bp downstream of the sgRNA) as well as all other captured 200 bp tiles with mean methylation level +/- 0.05 of the on-target locus in WT ES cells (collectively termed "offtarget"). Error bars display standard deviation for three clones. e Mean methylation for all CpGs within six 40 kb target regions that are covered in three independent clones over the Dox time course. Error bars display standard deviation for the three clones. $\mathbf{f}$ Venn diagram showing the overlap between the top 100 most highly methylated 200 bp tiles for the three independent clones

observed a decrease in the $\mathrm{BACH} 2$ target methylation within a few days post-transfection (Supplementary Fig. 5 j) $)^{13,27}$. Similarly, most off-target methylation decreased within 7 days posttransfection (Supplementary Fig. 5k), which is somewhat surprising given the low expression levels of TET enzymes (Supplementary Fig. 5l). This makes an active mechanism less likely and raises important questions regarding the stability of DNA methylation when it is added out of its normal context ${ }^{2}$.

dCas9-cat3a activity has limited impact on gene expression. To further investigate what renders loci more or less susceptible to off-target methylation, and considering that the majority of hypermethylated CGIs were located in promoter regions (Supplementary Fig. 6a), we wanted to explore how gained promoter methylation is linked to associated gene expression. We collected matched RNA samples from our $293 \mathrm{~T}$ cells before and after transfection and performed RNA-sequencing which confirmed the high abundance of cat3a transcripts that should originate from the exogenous dCas9-cat3a, stable levels of DNMT1 and background levels of DNMT3B (Supplementary Fig. 6b). Importantly, the dCas9-cat3a complex predominantly added methylation to promoters of either silenced or lowly expressed genes (Supplementary Fig. 6c, d) and was only retained at a subset of lowly transcribed genes after 7 days post-transfection (Supplementary Fig. 6c, Supplementary Data 1). Despite the gain of methylation, the vast majority of hypermethylated targets were not associated with significant changes in gene expression in the steady state (Supplementary Fig. 6e). In line with the fact that DNA methylation rarely acts as a primary silencing mechanism, our results suggest that silencing actively expressed genes using 
a

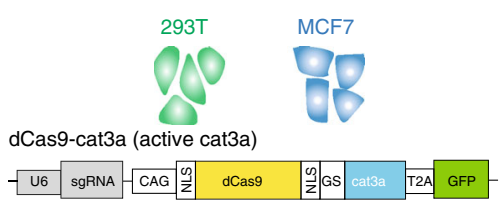

dCas9-cat3a-ANV (inactive cat3a)

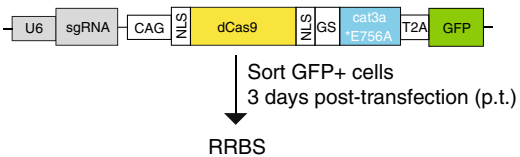

b

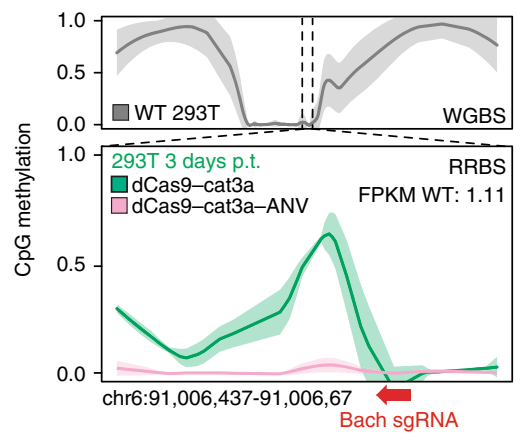

C

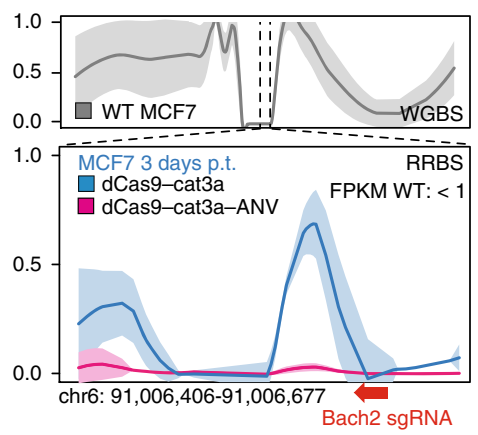

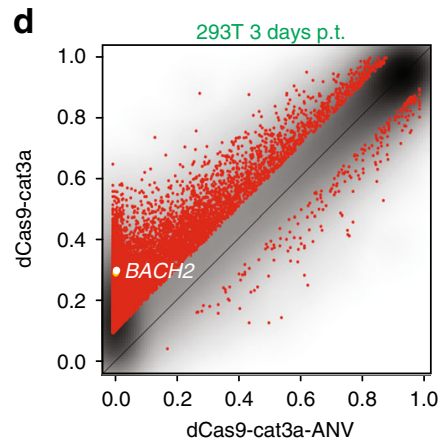

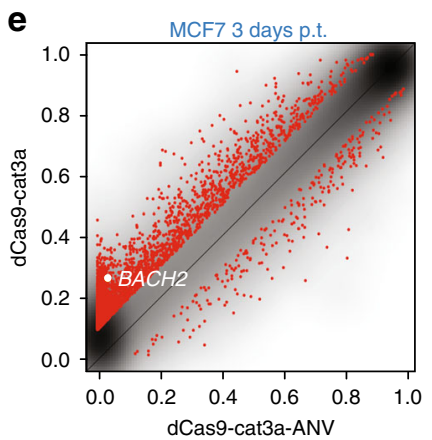

f

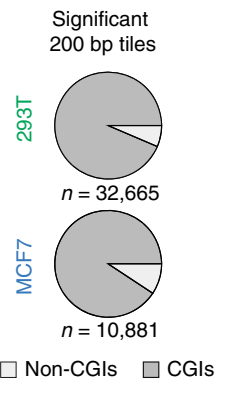

g

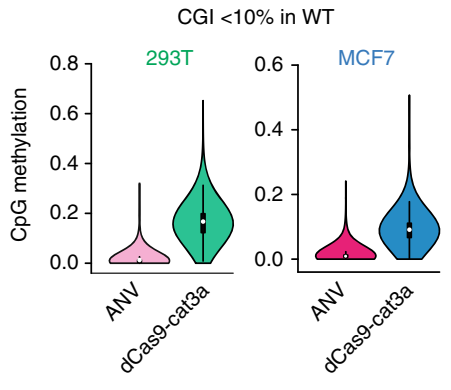

Fig. 3 Global off-target CpG methylation after transient dCas9-cat3a transfection. a 293T human embryonic kidney and MCF7 human breast cancer cells were transfected with pdCas9-DNMT3A-EGFP (Addgene \#71666) or pdCas9-DNMT3A-EGFP (ANV) (Addgene \#71685) with an sgRNA targeting $B A C H 2$. Cells were collected 3 days post-transfection (p.t.) and RRBS was performed. b Top: loess-smoothed CpG methylation for 293T cells (WGBS) highlighting the location of the BACH2 target within a CpG island. Bottom: the on-target smoothed methylation levels (RRBS) for 293T cells transfected with dCas9-cat3a or dCas9-cat3a-ANV 3 days p.t. The bold line indicates the mean methylation level with standard deviation shown by shaded fill. The position and orientation of the Bach2 sgRNA is indicated with a red arrow. c same as in b but for MCF7 cells. d Smooth scatter plot displaying mean methylation over 200 bp tiles in dCas9-cat3a and dCas9-cat3a-ANV transfected 293T cells 3 days p.t. with the Bach2 sgRNA (total tiles: 219,949). Red dots indicate tiles with significant changes in methylation (adj. $p$-value $<0.05$ ( $t$-test) and difference $>10 \%$, significant tiles: 32,665 ). The $B A C H 2$ locus is significant and highlighted as a white dot. e Same as in d but for MCF7 cells (total tiles: 200,157; significant tiles: 10,881). f The proportion of significantly differentially methylated 200 bp tiles that are located within CpG islands (CGIs) for 293T and MCF7 cells. $\mathbf{g}$ Methylation levels for CGls that have <10\% methylation in WT cells shown for dCas9-cat3a-ANV and dCas9-cat3a transfected 293T/MCF7 cells

dCas9-cat3a is not trivial and may require recruitment of additional repressive effectors ${ }^{10,28}$.

\section{Discussion}

Since the emergence of the CRISPR-Cas9 system for genome editing, it has evolved into a versatile toolbox for numerous applications, including the targeted manipulation of the epigenome using dCas9-coupled epigenetic effector proteins. Initial proof-of-concept studies have highlighted the general applicability of the system, thereby raising the possibility for functional dissection of individual epigenetic features and their direct impact on gene regulation ${ }^{8}$. However, this requires precise activity and to date a careful evaluation of the global on- vs. off-target activity has not been reported. Our study offers a unique tool (the $\mathrm{DKO}^{\text {zero }}$ line) to track all dCas9-methyltransferase activity that can be cleanly separated from any endogenous de novo methylation by Dnmt3a and Dnmt3b and therefore provides a first true account of the unexpected genome-wide DNA methylation footprints left by dCas9-coupled to the catalytic domain of Dnmt3a. We found that wild-type ES cells appear less susceptible to CGI off-target methylation than their somatic counterparts, which may in part be explained by the more frequent enrichment of $\mathrm{H} 3 \mathrm{~K} 4$ methylation at CGIs in pluripotent cells and also the higher levels of Tet expression (Supplementary Fig. 5l) ${ }^{29,30}$. Importantly, while sgRNAs can direct $\mathrm{CpG}$ methylation to targets in certain contexts, global off-target activity appears largely unaffected by the sgRNA presence, occurs regardless of experimental-setup (transfection vs. transduction) and across multiple cell types. This extensive off-target activity is perhaps not surprising considering that the amount of free nuclear dCas9cat3a is much higher than the target bound complex as each cell has only two alleles for a single copy locus that match the sgRNA. In the future, it would be interesting to explore whether redirecting the complex to inert genomic loci, such as abundant silent repeat elements or other ectopic targets would have an impact on reducing off-target activity by skewing its nuclear activity sufficiently to maximize the on- to off-target ratio. Furthermore, it remains to be seen whether other epigenetic effector fusions show similar global effects, although regulators of de novo DNA methylation may be particularly prone, as even transient interactions result in the addition of methyl groups that can subsequently be mitotically inherited through ubiquitously expressed DNMT1. Although we and others also highlight the transient nature of transfection-based methylation gain ${ }^{10,13}$, which will require further studies to delineate the underlying mechanism of failed, incomplete maintenance or active clearance. Nonetheless, sustained dCas9-cat3a activity may result in more permanent changes and it is worth considering the possibility that in some cases the aberrantly methylated loci could create a selective advantage that results in an undesired clonal expansion. Moreover, while we observed only limited effects on gene expression, we envision that these may be more striking in a system where cells are induced to change identity and have to activate previously silent regulators. In summary, our findings and the 
possible implications highlight the need to further investigate these valuable tools as well as remind us to consider the ubiquitous activity of dCas9-fused epigenetic effector proteins in experimental designs and interpretations.

\section{Methods}

dCas9-cat3a plasmid design and construction. We modified the dCas9 sequence of the pcDNA-dCas9 plasmid (Addgene \#47106) to include a BsaI restriction site followed by EcoRI and a Kozak sequence (N-terminus), as well as a BsiWI restriction site, P2A-GFP, followed by EcoRI and BsaI restriction sites (C-terminus). The C-terminal Nuclear Localization Sequence (NLS) was removed, the Nterminal NLS was left intact. Following BsaI digest, the dCas9-BsiWi-P2A-GFP fragment was subcloned into the FUW backbone (Addgene \#20723). The BsiWI restriction site was used to clone in the catalytic domain of Dnmt3a (cat3a, last 301 amino acids of Dnmt3a Isoform 1), with addition of a two amino acid linker (GS) between dCas 9 and cat3a.

LentiGuide-Puro cloning and transduction. The LentiGuide-Puro plasmid (Addgene \#52963) was used for downstream sgRNA integration. sgRNAs were subcloned using Gibson Assembly (see also: Research Gate DOI: 10.13140/ RG.2.1.1941.4161). Lentiviral particles were produced and the reset DKO ${ }^{\mathrm{dCas} 9-c a t 3 a}$ line was transduced with either a single sgRNA (Dazl) or a pool of 10 sgRNAs (Dazl, Dazl_2, Foxb1, FoxA2, H19, Fbxo40, Id3, Prdm14, Dppa3, and Pou5f1) for the hybrid capture experiment.

sgRNA sequences and coordinates. Mouse

Dazl: 5'-GAGGCGTGGGCTGCGCGCCC-3' (mm9, chr17:

50432925-50432944)

Dazl_2: 5'-GACGGGGCAGCTACGTGAGG-3' (mm9, chr17: 50432789-50432808)

Foxb1: 5'-GAAGGTAGAATGGGCAAGTC-3' (mm9, chr9: 69606961-69606980)

FoxA2: 5'-GTTTTAGTTACGAAATGCTT-3' (mm9,

chr2:147872854-147872873)

H19: 5'-GGAGACTGGGTGACCACGAG-3' (mm9, chr7: 149762698-149762717)

Fbxo40: 5'-GTACATCCCAAGTAGGCTAG-3' (mm9, chr16: 36969271-36969290)

Id3: 5'-GCGCCTGCGGGAACTGGTGC-3' (mm9, chr4:

135699940-135699959)

Prdm14: 5'-GAAGAATATGGATCCGGAGG-3' (mm9, chr1

13108873-13108892)

Dppa3: 5'-GGACAGATCCTGAGGGCTCA-3' (mm9, chr6:

122574622-122574641)

Pou5f1: 5'-GTGTCTTCCAGACGGAGGTT-3' (mm9: chr17:

35642849-35642868)

Human

Bach2: 5'-GAATGTAGCGATTGAGAGTGT-3' (hg19,

chr6:91006602-91006621)

Confirming gRNA targeting efficiency. To confirm the gRNA integration and targeting efficiency we nucleofected cells with pSpCas9(BB)-2A-GFP (Addgene \#PX458) and sorted for GFP 3 days post-nucleofection. Individual clones were picked and the targeting locus (Dazl) was amplified followed by sequencing.

Hybrid capture and Amplicon-Seq. The reset DKO ${ }^{\mathrm{dCas} 9-\mathrm{cat} 3 \mathrm{a}}$ line was transduced with a pool a 10 sgRNAs (see also section on sgRNA cloning, sequences and coordinates). Upon Puromycin selection $(2 \mu \mathrm{g} / \mathrm{ml})$, several colonies were picked, individually propagated and then induced for $0 \mathrm{~h}, 1$ day, 2 days and 3 days with doxycycline $(2 \mu \mathrm{g} / \mathrm{ml})$. Genomic DNA was isolated, followed by Amplicon-Seq to confirm the sgRNA composition in each clone. To do so, a fragment spanning the sgRNA integration site within the LentiGuide plasmid was amplified from the gDNA of each clonal line (FP: 5'-CGTGACGTAGAAAGTAATAATTTCTTG-3', RP: 5'-GTTATCAACTTGAAAAAGTGGCACCG-3'). Amplicons were repaired using the End-It DNA End-Repair Kit (Epicentre), extended using Klenow fragment ( $3^{\prime}-5^{\prime}$ exo) (NEB), and ligated to sequencing adapter oligos. Each library was then PCR-amplified using PFU Ultra II Hotstart Master Mix (Agilent), followed by pooling and sequencing on the MiSeq System (Illumina). sgRNAs were considered integrated if positive read counts were observed in the Amplicon-Seq.

Three clones with the following sgRNAs integrated were chosen for subsequent hybrid capture-based targeted bisulfite sequencing (custom Agilent SureSelectXT Methyl-Seq kit):

Clone 1: Dazl, Dazl_2, Foxb1, Foxa2, H19, Fbxo40, Id3, and Dppa3

Clone 3: Dazl, Dazl_2, Foxb1, Foxa2, H19, Fbxo40, Id3, and Dppa3

Clone 6: Dazl, Dazl_2, Foxb1, Foxa2, H19, Fbxo40, Id3, Dppa3, and Prdm14

On-target sites were tiled $20 \mathrm{~kb}$ upstream and $20 \mathrm{~kb}$ downstream of the sgRNA sequence using the SureDesign Custom Design Tool (Agilent). Our capture set also includes several additional control regions, which were not considered in our downstream analysis. The genomic DNA was sonicated (Covaris), ligated to methylated adapters and hybrid-selected using the designed custom $0.5-2.9 \mathrm{Mb}$ SureSelect bait library (Covaris). All captured genomic DNA was bisulfited converted, PCR amplified and sequenced on the Illumina HiSeq 2500.

Cell culture. ES cells were cultured in regular media containing 15\% FBS, 1\% PEN/ STREP, $1 \%$ glutamine, $1 \%$ NEAA, and $10^{5} \mathrm{U}$ LIF. For ES cell maintenance, dishes were coated with $0.2 \%$ gelatin and irradiated DR4 mouse embryonic fibroblasts (MEFs) were plated as a confluent layer of feeder cells. ES cells were seeded in a density of 50,000 cells per well of a six-well plate and were split every 3-4 days. Doxycycline and Puromycin were used at a concentration of $2 \mu \mathrm{g} / \mathrm{ml}$. MCF7 cells (ATCC HTB-22) were grown in DMEM F-12 with 10\% FBS and 1\% PenicillinStreptomycin. 293Ts (ATCC CRL-3216) were grown in DMEM supplemented with $10 \%$ FBS, $1 \%$ glutamine and $1 \%$ Penicillin-Streptomycin.

DKO ${ }^{z e r o}$ cell line generation. For the generation of our $\mathrm{DKO}^{\text {zero }}$ line, we transduced the original DKO line ${ }^{19}$ with a previously described Dnmt1 shRNA cassette (termed TKO-like cells) ${ }^{20,31}$. After several passages the TKO-like cells expressing the shRNA was transiently transduced with Cre recombinase (Addgene \#24593) to remove the shRNA-GFP construct, followed by sorting for GFP negative cells. Several clones were picked and propagated individually. To further enable any downstream Puromycin selection, the DKO $\mathrm{Dero}^{\text {zero }}$ line was modified by disrupting its existing ${ }^{19}$ Puromycin resistance cassette (sgRNA: $5^{\prime}$-GAACTCTTCCTCACGCGCGT- $3^{\prime}$ ). Individual clones were picked and DKO ${ }^{\text {zero }}$ clone 15.1 was chosen for all further experiments.

DKOdCas9-cat3a cell line generation. For the generation of the inducible $\mathrm{DKO}^{\mathrm{d}}$ Cas9-cat3a line, 20,000 DKO ${ }^{\text {zero }}$ ES cells were transduced with FUW-dCas9-cat3a and FUW-M2rtTA (Addgene \#20342) and treated with doxycycline to allow GFP based FACS and isolation of individual GFP-positive colonies. Cells were taken off doxycycline for one passage prior to LentiGuide-Puro (Addgene \#52963) transduction, (integration of Dazl sgRNA, sequence: 5'-GAGGCGTGGGCTGCGC GCCC-3'). Upon transduction, cells were selected with Puromycin for one passage, after which dCas9-cat3a was induced for two passages followed by genomic DNA extraction and whole-genome bisulfite sequencing (WGBS). To reset the $\mathrm{DKO}^{\mathrm{dCas} 9-\text { cat3a }}$ line, cells were passaged in $2 \mathrm{i} / \mathrm{Vitamin} \mathrm{C}^{22}$ and $\mathrm{Dnmt1}$ was transiently knocked down using the shRNA described above. For the time course experiment cells were induced for 0,2 , or 7 days, followed by DNA extraction and WGBS. Homogenous induction was confirmed by inducing cells for three days with or without doxycycline, followed by FACS analysis.

DKO ${ }^{\text {cat3a }}$, DKO ${ }^{\text {Dnmt3a }}$ and $\mathbf{W T}^{\text {dCas9-cat3a }}$ generation. The full-length Dnmt3a Isoform 1 or its catalytic domain (the last 301 amino acids) were fused to P2AGFP, followed by subcloning into the FUW backbone (Addgene \#20723). DKO ${ }^{\text {zero }}$ cells were transduced with FUW-M2rtTA (Addgene \#20342) and FUW-Dnmt3a-GFP or FUW-cat3a-GFP, respectively and treated with doxycycline for about two weeks to allow GFP based FACS and isolation of individual GFP-positive colonies. For the generation of the inducible dCas9-cat3a wild-type line, V6.5 wild-type ES cells were transduced with FUW-M2rtTA and FUW-dCas9-cat3a, selected and induced as described above. Each cell line was induced with doxycycline for two passages (7 days) prior to DNA extraction and bisulfite sequencing. For the WT ${ }^{\text {dCas9-cat3a }}$ line, a non-induced (no doxycycline) sample was also collected for comparison.

Transfection experiments. MCF7 and 293T cells were transfected with pdCas9-DNMT3A-EGFP (Addgene \#71666) or pdCas9-DNMT3A-EGFP-ANV (Addgene \#71685) including the Bach2 sgRNA (sequence: $5^{\prime}$-AATGTAGCGATTGAGAGTGT-3', see also ref. 13) using FUGENE HD Transfection Reagent (Promega). Cells were sorted for GFP 3 days post-transfection if transfection efficiency was below $90 \%$ (based on visual inspection). DKO ${ }^{\text {zero }}$ cells were transfected with pdCas9-DNMT3A-EGFP (Addgene \#71666) or pdCas9-DNMT3AEGFP-ANV (Addgene \#71685) including the Dazl sgRNA (sequence: 5'-GAGG CGTGGGCTGCGCGCCC-3') using Xfect mouse ES cell Transfection reagent (Clontech). 293T cells were partially replated 3 days post-transfection for later collection time points at day 7 and day 15. Upon DNA extraction, the DNA was digested with DpnI for $2 \mathrm{~h}$ at $37^{\circ} \mathrm{C}$ to digest all residual plasmid DNA. This was followed by a $0.4 \times$ AMPure XP bead (Beckman Coulter) cleanup to select for high molecular/non-digested DNA.

RNA-sequencing. RNA-seq libraries were prepared using the TrueSeq RNA Sample Prep v2 HS Protocol (Illumina), followed by $50 \mathrm{bp}$ paired-end sequencing on the NextSeq 500 Sequencing System (Illumina).

Reduced representation bisulfite sequencing. Genomic DNA was quantified using a Qubit 2.0 Fluorometer, and quality-assessed on an Agilent 2200 TapeStation D1000 ScreenTape. RRBS was performed on $20 \mathrm{ng}$ of each sample using the NuGen Ovation ${ }^{\circledR}$ RRBS Methyl-Seq System following the manufacturer's recommendations except that barcoded adapter-ligated samples were pooled in groups of 
8 immediately prior to bisulfite conversion with the Qiagen EpiTect Fast Bisulfite Conversion kit. Library pools were purified with a $1 \times$ Agencourt RNA XP bead cleanup and sequenced on the HiSeq 2500 or NextSeq 500 Sequencing System (Illumina). Sequenced reads were aligned to the $\mathrm{mm} 9$ or hg19 reference genome using BSmap ${ }^{32}$. Custom scripts were used to determine whether cytosines were methylated or unmethylated by observing bisulfite conversion of unmethylated cytosines in comparison to the reference sequence.

WGBS library construction and processing. 100-200 ng of genomic DNA was fragmented using a Covaris S2 for 6 min according to the following program: duty cycle $5 \%$; intensity 10 ; cycle per burst 200 . The sheared DNA was purified using the DNA Clean and Concentrator kit from Zymo. Bisulfite conversion of DNA was then conducted using the EZ DNA Methylation-Gold kit (Zymo Research), eluting in $15 \mu \mathrm{l}$ low TE buffer. To minimize loss during storage, bisulfite-converted DNA was immediately processed for generating WGBS libraries using the Accel-NGS Methyl-Seq DNA library kit (Swift Biosciences). All protocols were carried out according to manufacturer's recommendations unless specified. The libraries were sequenced with 100 bp paired-end reads on an Illumina HiSeq 2500 sequencer. 10 bp were clipped from the WGBS raw sequencing reads at either end. The reads were then aligned to the $\mathrm{mm} 9$ build of the mouse genome using BSmap ${ }^{32}$. DNA methylation calling for individual $\mathrm{CpG}$ sites was performed using mcall from the MOABS package ${ }^{33}$. CpA calling was performed using mcall with setting adjusted to detect CpAs.

Data processing and analysis. For all methylation analysis, only $\mathrm{CpGs}$ covered by at least 5 reads were considered (unless otherwise stated). For genomic methylation plots, loess smoothing and standard deviation calculations were derived using the msir package in R (V1.3.1) with span set to 0.4. Violin plots were created using the $\mathrm{R}$ package 'vioplot' using standard parameters. Boxplots and heatmaps were created using R.

For all genomic feature comparisons, $\mathrm{CpGs}$ were intersected using BedTools v2.25.0 with: CpG islands, as previously defined ${ }^{34}$ or exons, introns, CGI shores, short interspersed nuclear repeats (SINEs), long interspersed nuclear repeats (LINEs), long terminal repeats (LTRs) or Intra-cisternal A-type particle (IAP) elements downloaded from the UCSC Genome Browser ( $\mathrm{mm} 9$ or hg19 depending on data).

For the hybrid capture data, the methylation bed files were intersected with the six $40 \mathrm{~kb}$ target regions. IGV tracks were created with all CpGs covered by at least 5 reads. To compare on-target vs. off-target methylation, we compared the methylation level of the $200 \mathrm{bp}$ immediately downstream of the sgRNA sequence (on-target), to all captured $200 \mathrm{bp}$ tiles with the same methylation level in WT cells (i.e., with the same potential to become methylated; off-target). The difference between 0 day and 3 day mean methylation was calculated for the three clones including mean and standard deviation. To compare the most highly methylated tiles between clones, we selected the top 100 methylated 200 bp tiles in each sample and computed the overlap, plotting venn diagrams manually.

To generate delta methylation histograms for WT vs. WT ${ }^{\mathrm{dCas} 9-\mathrm{cat3a}}$ cells, we compared mean methylation over $1 \mathrm{~kb}$ tiles.

To compare methylation levels between transfected samples, we tiled the genome with $200 \mathrm{bp}$ tiles and performed paired $t$-tests for CpGs with $\geq 10$ reads within the tile calculating $p$-values adjusted using the Benjamini-Hochberg model. Only tiles containing at least $3 \mathrm{CpGs}$ were considered. Significant tiles had adj. $p$ value $<0.05$ and at least $10 \%$ difference in methylation compared to WT.

To identify significant changes in CGI methylation, the same $t$-test was performed over each CGI (with $\geq 3 \mathrm{CpGs}$ ) with significant islands selected as adj. $p$ value $<0.05$ with no cutoff for change in methylation.

To compare CGI methylation to H3K4me3 methylation, BedTools was used to obtain read counts per CpG island. The number of reads was then divided by the length of the CGI with the top 1\% values normalized to 100 and the rest of the values scaled accordingly.

To analyze individual reads, custom perl scrips were used to extract per CpG methylation levels across reads. Reads were then plotted using $\mathrm{R}$ for individual loci to study examples of per-read $\mathrm{CpG}$ methylation. Seperately, all reads located within $200 \mathrm{bp}$ windows where CpGs showed significant changes in methylation were analyzed to determine whether all reads or only a subset acquired methylation. Reads were allocated into the category "none" if no methylation existed, and "some" if we detected at least one methylated $\mathrm{CpG}$.

RNA-seq data was aligned using STAR aligner followed by stringtie v1.3 to the hg19 genome. FPKM values collapsed by gene were extracted and compared for two technical replicates. Gene-specific FPKM values were TMM normalized using edgeR in R. For differential analysis (log fold change), all transcripts with FPKM $<1$ were removed.

FPKM levels for Tet1 and Tet2 in mouse ES and human ES were taken from two previously published papers ${ }^{35,36}$.

Nuclear extraction and western blotting. Approximately six million ES cells (V6.5 and reset DKO ${ }^{\mathrm{dCas} 9-\mathrm{cat} 3 \mathrm{a}}$ ) were MEF depleted and resuspended in $7 \mathrm{ml}$ lysis buffer containing $25 \mathrm{mM}$ HEPES $\mathrm{pH}$ 7.6, $5 \mathrm{mM} \mathrm{MgCl} 2,25 \mathrm{mM} \mathrm{KCl}, 0.05 \mathrm{mM}$ EDTA, $10 \%$ Glycerol, $0.1 \%$ IGEPAL, $1 \mathrm{mM}$ DTT, $1 \times$ Roche proteinase inhibitor.
The lysate was centrifuged for $5 \mathrm{~min}$ at $211 \times g$ and the supernatant was discarded. The precipitate was resuspended in $500 \mu \mathrm{l}$ cold buffer containing $10 \mathrm{mM}$ HEPES pH7.6, $3 \mathrm{mM} \mathrm{MgCl}$, $100 \mathrm{mM} \mathrm{KCl}, 0.01 \mathrm{mM}$ EDTA, 10\% Glycerol, 1 mM DTT, $1 \times$ Roche proteinase inhibitor to wash nuclei, centrifuged $5 \mathrm{~min}$ at $3000 \times g$ and the supernatant was discarded. The precipitate was resuspended in $100 \mu \mathrm{l}$ RIPA buffer containing DNase and RNase, vortexed for $20 \mathrm{~min}$ at $4^{\circ} \mathrm{C}$, centrifuged for $15 \mathrm{~min}$ at $13,523 \times g$. Supernatant containing soluble proteins was transferred to a new tube, loading buffer (NuPAGE LDS Sample Buffer $4 \times$, Thermo Fisher Scientific) was added to $1 \times$ and denatured for $10 \mathrm{~min}$ at $70^{\circ} \mathrm{C} .15 \mu \mathrm{l}$ of the sample was run on a NuPage $4-12 \%$ Bis-Tris protein gel in $1 \times$ MOPS buffer with $10 \mu$ l of Precision Plus Protein Dual Color Standards ladder (Bio-Rad) for $1 \mathrm{~h}$ at $200 \mathrm{~V}$. Protein was then transferred on a nitrocellulose membrane by wet electroblotting for $2 \mathrm{~h}$ at 340 $\mathrm{mA}$. The membrane was blocked in 5\% non-fat milk, cut into two sections and incubated with mouse primary antibodies anti-DNMT1 (1:2000 Active Motif \#39204) and anti-Tubulin (1:10,000 DSHB \#12G10) overnight, washed three times for $10 \mathrm{~min}$ in PBS with $0.1 \%(\mathrm{v} / \mathrm{v})$ Tween and incubated with anti-mouse-HRP secondary antibody (1:10,000 Jackson Laboratory \#115-035-174) for $1 \mathrm{~h}$. Following three 10 min washing steps in PBS with $0.1 \%$ Tween, the protein bands were visualized using ECL reagent (GE Healthcare). Uncropped images of Fig. $1 \mathrm{~b}$ are available in Supplementary Fig. 1.

Data availability. All sequencing data that support the findings of this study have been deposited in the National Center for Biotechnology Information Gene Expression Omnibus (GEO) and are accessible through the GEO Series accession number GSE87757. Gene-specific FPKM values for the RNA-seq data is also available as Supplementary Data File 1. FPKM levels for Tet1 and Tet2 in mouse ES cells and human ES cells from two previously published papers ${ }^{35,36}$ are available from GEO under accession codes human ES cells: GSM1112834 and GSM1112837 and mouse ES cells: GSM1842768 and GSM1842769.

Received: 3 December 2017 Accepted: 20 December 2017

Published online: 09 February 2018

\section{References}

1. Bestor, T. H. The DNA methyltransferases of mammals. Hum. Mol. Genet. 9 2395-2402 (2000).

2. Smith, Z. D. \& Meissner, A. DNA methylation: roles in mammalian development. Nat. Rev. Genet. 14, 204-220 (2013).

3. Zemach, A., McDaniel, I. E., Silva, P. \& Zilberman, D. Genome-wide evolutionary analysis of eukaryotic DNA methylation. Science 328, 916-919 (2010).

4. Lister, R. et al. Human DNA methylomes at base resolution show widespread epigenomic differences. Nature 462, 315-322 (2009).

5. Stadler, M. B. et al. DNA-binding factors shape the mouse methylome at distal regulatory regions. Nature 480, 490-495 (2011).

6. Ziller, M. J. et al. Charting a dynamic DNA methylation landscape of the human genome. Nature 500, 477-481 (2013).

7. Shipony, Z. et al. Dynamic and static maintenance of epigenetic memory in pluripotent and somatic cells. Nature 513, 115-119 (2014).

8. Stricker, S. H., Koferle, A. \& Beck, S. From profiles to function in epigenomics. Nat. Rev. Genet. 18, 51-66 (2017).

9. Hsu, P. D., Lander, E. S. \& Zhang, F. Development and applications of CRISPRCas9 for genome engineering. Cell 157, 1262-1278 (2014).

10. Amabile, A. et al. Inheritable silencing of endogenous genes by hit-and-run targeted epigenetic editing. Cell 167, 219-232 (2016).

11. Kungulovski, G. \& Jeltsch, A. Epigenome editing: state of the art, concepts, and perspectives. Trends Genet. 32, 101-113 (2016).

12. Liu, X. S. et al. Editing DNA methylation in the mammalian genome. Cell 167, 233-247 (2016). e217.

13. Vojta, A. et al. Repurposing the CRISPR-Cas9 system for targeted DNA methylation. Nucleic Acids Res. 44, 5615-5628 (2016).

14. McDonald, J. I. et al. Reprogrammable CRISPR/Cas9-based system for inducing site-specific DNA methylation. Biol. Open 5, 866-874 (2016).

15. Stepper, P. et al. Efficient targeted DNA methylation with chimeric dCas9Dnmt3a-Dnmt3L methyltransferase. Nucleic Acids Res. 45, 1703-1713 (2017).

16. Huang, Y. H. et al. DNA epigenome editing using CRISPR-Cas SunTagdirected DNMT3A. Genome Biol. 18, 176 (2017).

17. Kungulovski, G. et al. Targeted epigenome editing of an endogenous locus with chromatin modifiers is not stably maintained. Epigenetics Chromatin 8, 12 (2015).

18. $\mathrm{Wu}, \mathrm{X}$. et al. Genome-wide binding of the CRISPR endonuclease Cas9 in mammalian cells. Nat. Biotechnol. 32, 670-676 (2014).

19. Okano, M., Bell, D. W., Haber, D. A. \& Li, E. DNA methyltransferases Dnmt3a and Dnmt3b are essential for de novo methylation and mammalian development. Cell 99, 247-257 (1999). 
20. Meissner, A. et al. Reduced representation bisulfite sequencing for comparative high-resolution DNA methylation analysis. Nucleic Acids Res. 33, 5868-5877 (2005).

21. Habibi, E. et al. Whole-genome bisulfite sequencing of two distinct interconvertible DNA methylomes of mouse embryonic stem cells. Cell Stem Cell 13, 360-369 (2013).

22. Blaschke, K. et al. Vitamin C induces Tet-dependent DNA demethylation and a blastocyst-like state in ES cells. Nature 500, 222-226 (2013).

23. Rose, N. R. \& Klose, R. J. Understanding the relationship between DNA methylation and histone lysine methylation. Biochim. Biophys. Acta 1839, 1362-1372 (2014)

24. Meissner, A. et al. Genome-scale DNA methylation maps of pluripotent and differentiated cells. Nature 454, 766-770 (2008).

25. Sternberg, S. H., Redding, S., Jinek, M., Greene, E. C. \& Doudna, J. A. DNA interrogation by the CRISPR RNA-guided endonuclease Cas9. Nature 507, 62-67 (2014).

26. Ziller, M. J. et al. Genomic distribution and inter-sample variation of non-CpG methylation across human cell types. PLoS Genet. 7, e1002389 (2011).

27. Ford, E.E. et al. Frequent lack of repressive capacity of promoter DNA methylation identified through genome-wide epigenomic manipulation. bioRxiv https://doi.org/10.1101/170506 (2017).

28. Smith, Z. D., Sindhu, C. \& Meissner, A. Molecular features of cellular reprogramming and development. Nat. Rev. Mol. Cell. Biol. 17, 139-154 (2016).

29. Mikkelsen, T. S. et al. Genome-wide maps of chromatin state in pluripotent and lineage-committed cells. Nature 448, 553-560 (2007).

30. Koh, K. P. et al. Tet 1 and Tet 2 regulate 5-hydroxymethylcytosine production and cell lineage specification in mouse embryonic stem cells. Cell Stem Cell $\mathbf{8}$, 200-213 (2011).

31. Ventura, A. et al. Cre-lox-regulated conditional RNA interference from transgenes. Proc. Natl Acad. Sci. USA 101, 10380-10385 (2004).

32. Xi, Y. \& Li, W. BSMAP: whole genome bisulfite sequence MAPping program. BMC Bioinformatics 10, 232 (2009).

33. Sun, D. et al. MOABS: model based analysis of bisulfite sequencing data Genome Biol. 15, R38 (2014).

34. Illingworth, R. et al. A novel CpG island set identifies tissue-specific methylation at developmental gene loci. PLoS Biol. 6, e22 (2008).

35. Gifford, C. A. et al. Transcriptional and epigenetic dynamics during specification of human embryonic stem cells. Cell 153, 1149-1163 (2013).

36. Galonska, C., Ziller, M. J., Karnik, R. \& Meissner, A. Ground state conditions induce rapid reorganization of core pluripotency factor binding before global epigenetic reprogramming. Cell Stem Cell 17, 462-470 (2015).

\section{Acknowledgements}

We thank Stefanie Grosswendt, Rahul Karnik and members of the Meissner lab for their help and in particular Zachary D Smith for critical discussions. A.L.M. is supported by the National Science Foundation Graduate Research Fellowship. A.M. is a New York Stem Cell Foundation, Robertson Investigator. This work was supported by NIH grant R01DA036898, The Starr Foundation, the New York Stem Cell Foundation and the Max Planck Society.

\section{Author contributions}

C.G. and A.M. designed and conceived the study, C.G. generated the constructs, cell lines and performed the experiments, J.C. performed the data analysis, C.G., J.C., and A.M. interpreted the data, C.G. and A.L.M. did the full-length overexpression, J.D. and D.C. helped with the derivation of the inducible lines, E.K.S., H.G., A.W.M., and A.G. generated the DNA methylation profiling libraries, C.G. and M.J.Z. designed the hybrid capture probe set, S.K. and B.T. performed the sequencing and initial data processing, K. C. provided support with analysis strategy. T.C. and H.R.S. provided support with interpretation of the data. C.G., A.M., and J.C. wrote the manuscript.

\section{Additional information}

Supplementary Information accompanies this paper at https://doi.org/10.1038/s41467 017-02708-5.

Competing interests: The authors declare no competing financial interests.

Reprints and permission information is available online at http://npg.nature.com/ reprintsandpermissions/

Publisher's note: Springer Nature remains neutral with regard to jurisdictional claims in published maps and institutional affiliations.

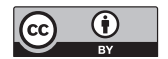

Open Access This article is licensed under a Creative Commons Attribution 4.0 International License, which permits use, sharing, adaptation, distribution and reproduction in any medium or format, as long as you give appropriate credit to the original author(s) and the source, provide a link to the Creative Commons license, and indicate if changes were made. The images or other third party material in this article are included in the article's Creative Commons license, unless indicated otherwise in a credit line to the material. If material is not included in the article's Creative Commons license and your intended use is not permitted by statutory regulation or exceeds the permitted use, you will need to obtain permission directly from the copyright holder. To view a copy of this license, visit http://creativecommons.org/ licenses/by/4.0/.

(c) The Author(s) 2018 